\title{
The need for hypoxic exposure in experimental PAH - Comment on Chen et al.: a novel rat model of pulmonary hypertension induced by mono treatment with SU5416
}

\author{
Michiel Alexander de Raaf $^{1} \cdot$ Ingrid Schalij $^{1} \cdot$ Harm Jan Bogaard ${ }^{1}$
}

Received: 28 July 2020 / Revised: 3 August 2020 / Accepted: 5 August 2020 / Published online: 4 September 2020

(c) The Japanese Society of Hypertension 2020

We read with great interest the report by Chen et al. on a novel rat model of experimental pulmonary hypertension (expPH) based on a single injection of SU5416 (Sugenonly) in adult rats [1]. While VEGF-R inhibition has already been shown to induce mild hypertension and emphysematous lung changes in neonatal rats some two decades ago [2], the novelty of Chen's findings is that even in older rats, a single dose of SU5416 can induce significant pulmonary vascular remodeling, pulmonary hypertension and signs of right ventricular (RV) failure. While these findings were milder than those in the well-known expPH model based on a combination of SU5416 with hypoxia (SuHx model), Chen et al. argue that their model of expPH may provide new insights in studying the subclinical phase of pulmonary arterial hypertension.

Because PAH patients are not exposed to hypoxia, Chen et al. conclude that the Sugen-only model is a more appropriate model for resembling PAH than the SuHx model. Here, we would like to argue that this conclusion may be premature. First, the description given by Chen et al. of lung histology in the Sugen-only model is not very detailed. Importantly, the degree of emphysematous changes is not described, and there is no mention of occlusive intimal changes, findings that are very important in human $\mathrm{PAH}$. Second, the described degree of RV failure in this model seems out of proportion to the degree of pulmonary vascular remodeling. Cardiac output in Sugen-only rats was reduced to almost the same degree as in SuHx rats, which is not expected for such a mild degree of pressure overload.

Harm Jan Bogaard

hj.bogaard@amsterdamumc.nl

1 Amsterdam UMC, Vrije Universiteit Amsterdam, Department of Pulmonology, Amsterdam Cardiovascular Sciences, $1081 \mathrm{HV}$ Amsterdam, The Netherlands
We think that it is premature to state the translational superiority of this model for PAH.

We previously showed that SuHx rats initially recover from severe pulmonary hypertension once they are reexposed to room air. After cessation of hypoxia, reversible hypoxic vasoconstriction is abolished and blood viscosity normalizes due to a decreasing hematocrit. Subsequently, over the course of several weeks, the degree of pressure overload rises as pulmonary vascular remodeling progresses even without exposure to hypoxia [3]. Ultimately, this leads to plexiform-like lesions in the lung, a pathognomonic finding in the human PAH lung [4]. Therefore, in Chen's study, the Sugen-only model is compared to the SuHxmodel at its 'weakest moment'. It is unfortunate that the article by Chen et al. does not include a histological comparison of both models several weeks after hypoxic exposure in SuHx rats. In this way, the degree of emphysema could have been compared, as it is still controversial whether emphysema is important in SuHx rats [5, 6].

When choosing an animal model to mimic pulmonary hypertension, the decision should be guided by the most pertinent research question at hand: when does the model match the patient [7]? When searching for new treatments for classical severe PAH, it is probably still preferable to use the SuHx rat model. When studying PH in the context of emphysema and perhaps milder forms of $\mathrm{PH}$, the Sugenonly model may be of additive value. There is still quite some characterization to be done, however, before the use of this model can truly be advocated.

\section{Compliance with ethical standards}

Conflict of interest The authors declare that they have no conflict of interest.

Publisher's note Springer Nature remains neutral with regard to jurisdictional claims in published maps and institutional affiliations. 


\section{References}

1. Chen Y, Kuang M, Liu S, Hou C, Duan X, Yang K, et al. A novel rat model of pulmonary hypertension induced by mono treatment with SU5416. Hypertens Res. 2020;43:754-64.

2. Le Cras TD, Markham NE, Tuder RM, Voelkel NF, Abman SH. Treatment of newborn rats with a VEGF receptor inhibitor causes pulmonary hypertension and abnormal lung structure. Am J Physiol Lung Cell Mol Physiol. 2002;283:L555-62.

3. de Raaf MA, Schalij I, Gomez-Arroyo J, Rol N, Happé C, de Man FS, et al. SuHx rat model: partly reversible pulmonary hypertension and progressive intima obstruction. Eur Respir J. 2014;44:160-8.

4. Abe K, Toba M, Alzoubi A, Ito M, Fagan KA, Cool CD, et al. Formation of plexiform lesions in experimental severe pulmonary arterial hypertension. Circulation. 2010;121:2747-54.
5. Kojonazarov B, Hadzic S, Ghofrani HA, Grimminger F, Seeger W, Weissmann N, et al. Severe emphysema in the SU5416/hypoxia rat model of pulmonary hypertension. Am J Respir Crit Care Med. 2019;200:515-8.

6. Bogaard HJ, Legchenko E, Chaudhary KR, Sun XQ, Stewart DJ, Hansmann G. Emphysema is-at the most-only a mild phenotype in the sugen/hypoxia rat model of pulmonary arterial hypertension. Am J Respir Crit Care Med. 2019;200:1447-50.

7. Happé C, Kurakula K, Sun X-Q, da Silva Goncalves Bos D, Rol N, Guignabert $\mathrm{C}$, et al. The BMP receptor 2 in pulmonary arterial hypertension: when and where the animal model matches the patient. Cells. 2020;9:1422. 